\title{
An Energy Efficient and Lifetime Ratio Improvement Methods Based on Energy Balancing
}

\author{
Niranjan L., Manoj Priyatham M.
}

\begin{abstract}
WSN consist of set of Sensing points which are responsible for collecting the detected information and then send the packets towards control centre which is responsible for processing of data. The applications of WSN include environmental data analysis, defence data collection and information. The survey of algorithms is done for the improvement of lifetime ratio. Four different algorithms namely Random, Random-CGT, EGT-Random and GTEB algorithms. The four algorithms are compared and then it is proved GTEB exhibits best behaviour with respect to energy consumed, number of non-holes, number of holes, Non-Hole to Hole ratio, residual energy, overhead and throughput.
\end{abstract}

Keywords- Wireless Sensor Sensing points, Global Energy Balance, Energy Consumption.

\section{INTRODUCTION}

WSN is used to sparse the Sensing points in the rectangular area. Each and every Sensing Point will have its own unique positional coordinate and a unique identifier. The positional coordinate is a combination of (xp, yp) along with a unique identification number.

The network classification is scaled into two kinds Single Area Network and Multi Area Network. For a Single Area Network all the Sensing points are spread in the single co-existing unit. The network can be treated as autonomous with no control as seen in Fig.1. The Single System network as shown in the Fig. 1 has 100 Sensing points which are spread in an area of $100 * 100 \mathrm{~m}$. Sensing Point 9 has been placed at the coordinate $(9,11)$. Sensing Point 37 has been placed at the coordinate $(42,2)$ and Sensing Point 20 has been placed at the coordinate $(79,1)$ and Sensing Point 52 has been placed at the coordinate $(90,10)$.
Revised Manuscript Received on December 15, 2019

Mr. Niranjan L, Research Scholar, Department of Electronics and Communication Engineering, A P S College of Engineering, Bengaluru, India.niranjan11983@gmail.com

Dr. Manoj Priyatham M, Professor, Department of Electronics and Communication Engineering, A P S College of Engineering, Bengaluru, India.drmmp777@gmail.com

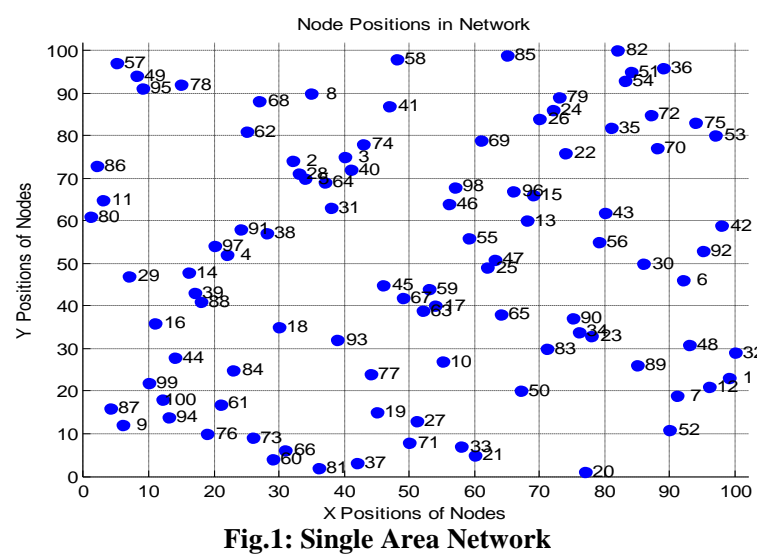

Multi Area Network is the network in which the Sensing points will be displaced across multiple units in a positional space. Each system will have set of Sensing points. In this kind of network there are 2 types of communication which are possible one is Inter System and another one is Intra System communication. For Intra System Communication the communication happens between the Sensing points within the same System. For Inter System communication between the Sensing Point in one System to a Sensing Point in a different System. Multi System Network can be defined as shown in Fig. 2. The Fig. 2 shows the multi area network. As shown in the Fig. 2 there are four different independent units. The first unit has 10 Sensing points, the second unit has 5 Sensing points, the third unit has another 10 Sensing points and finally fourth unit has another 10 Sensing points.

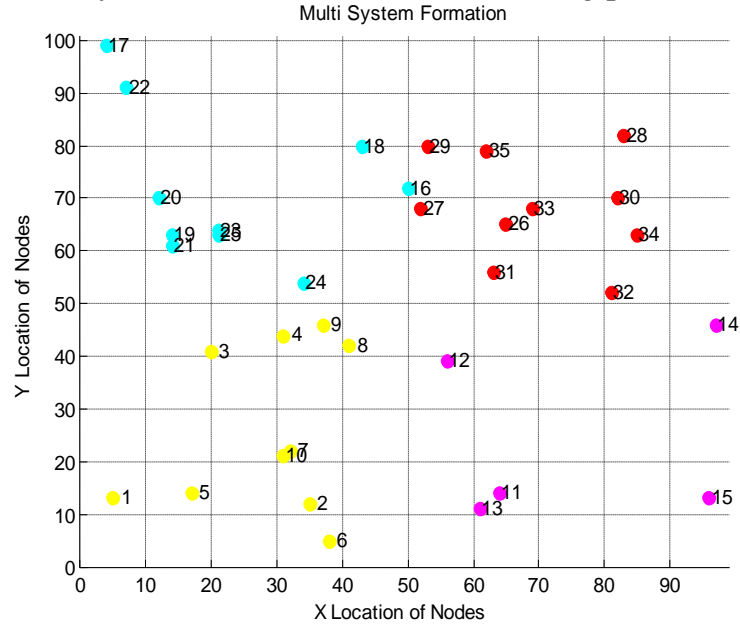

Fig. 2: Multi System Network 
Few applications of WSN network can be summarized as given in the Fig. 3. Defence applications, Environmental Monitoring applications, Logistics applications, Hospital \& Smart health Clinics applications, Transport Device applications. The WSN is widely applicable for most of the devices which are working in wireless environment. The Fig. 3 shows the application of WSN in various streams.

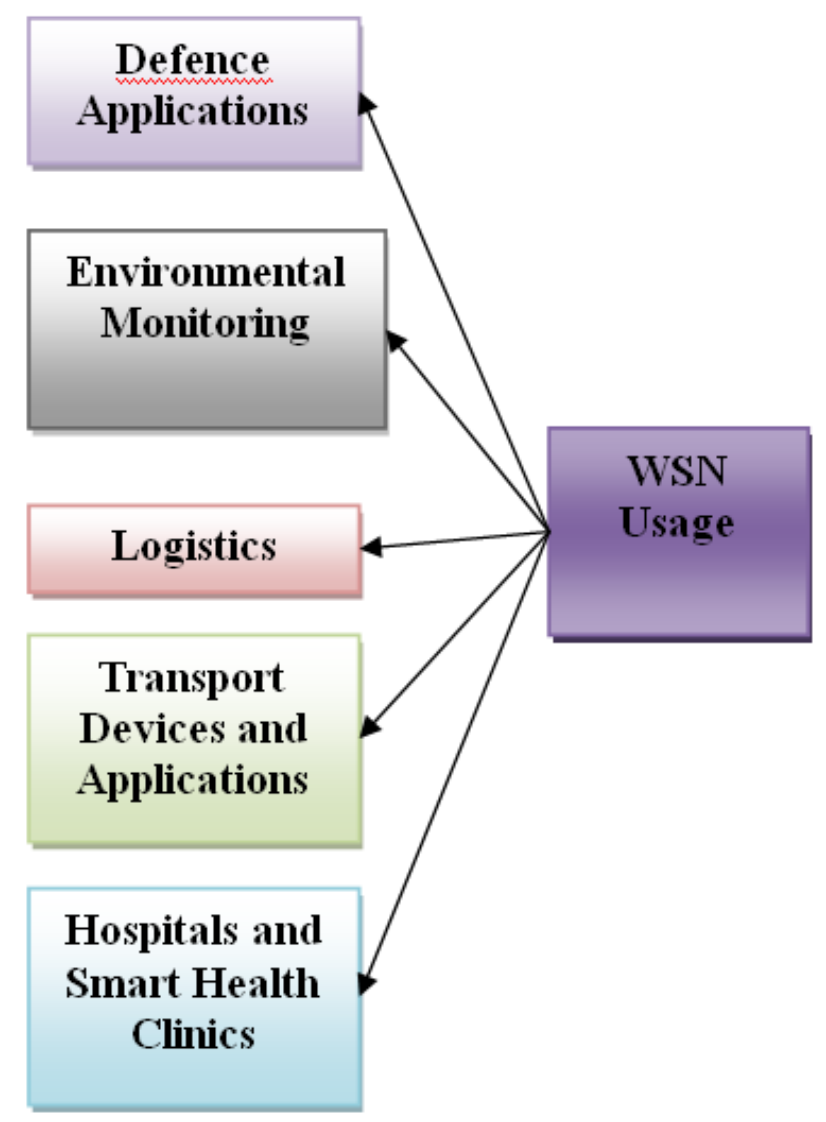

Fig. 3: Applications of WSN

WSN has been used for wide variety of applications beginning from 1980s. The most important limitation of WSN Sensing Point is battery capacity. WSN can be used for different kind of applications. It can be used for applications ranging from Defence Applications, Environment Monitoring, Logistics, Transportation and Hospital Management

The rest of the explanation is divided as follows. Section 2 will have described the Survey of Game Theory based Energy Efficiency and Lifetime Improvement techniques. Section 3 will describe four existing methods of Game Theory. Section 4 provides the simulation results of Game Theory Energy Balancing Method (GTEB) along with that the comparison performed with respect to Random, CGT-Random, EGT-Random for various parameters.

\section{LITERAURE SURVEY}

The load of packets in the network can be balanced with the help of game theoretic based methods. The major aim is to have a method which can allow the Sensing Point to lose their energy approximately at the same level. The three-dimensional game theory based energy balancing will combine multiple methods to have better decisions while choosing the forwarding Sensing points by performing

balanced energy reduction and selecting a proper forward Sensing Point [1].

The region around the initiator Sensing Point is divided into multiple sections by computing the density value of packets residing in the network. The regions are selected based on remaining packets in the section and then one of the Sensing Point in the section is picked up to send the packet. This approach increases the delivery parameter and lifetime parameter [2]

The sensing units are randomly spread in a System and then send the analysed data to the receiver agent. From each of the System a special agent is chosen based on energy balancing to communicate within and between Systems in an efficient fashion based on fuzzy method [3].

The entire area is spread with sensor Sensing points which have various characteristics like low power, short lifetime and sometimes unreliability. The main constraint is to scale up lifetime ratio of the network so that the network coverage is more. The probability value is computed and then scheduler is executed in an optimized fashion to deliver the packets with low overhead ratio and better coverage scale [4].

When the data aggregation is performed in the network there is generally large amount of energy wastage to maintain the security of data. The entire network is subdivided into System of trees; one tree is connected to other tree with the help of chains. The optimization of energy efficiency is achieved with the help of tail Sensing points present in the chains. The method decreases the amount of energy wastage and helps in increasing the lifetime ratio [5].

The data must be transmitted to the sink at regular intervals of time "Ti". The table of the sensors have to be maintained which can have path in the packet structure. The value of data size along with speed at which data is acquired is taken to provide different frequency for each of the Sensing points so that aggregation of information can be done with less energy consumption and improving the lifetime ratio of the network [6].

The social media, news, devices, monitoring of places produces huge amount of data. Internet of things (IoT) along with wireless sensor networks (WSNs) help in collecting data, execute analysis and then provide recommendations to the end user. The redundancy of the data across multiple Sensing points is a novel research task. The prediction model is triggered in a round robin principle with respect to lifetime parameter.

Each round is defined as a set of periods $\mathrm{T} 1, \mathrm{~T} 2, \ldots \mathrm{T}$. The rounds T1 to Tn are divided into subset, Sensing points will collect data only on those subset times, then forward data to next Sensing points before entering the sleep mode. The sink executes long short-term memory (LSTM) along with time series model to find the predicted data during sleep mode. This will help in amount of transmission done by a Sensing Point and hence improves lifetime ratio [7].

There are multiple Sensing points which are distributed under various Systems in wireless sensor network. Two techniques when combined can help in effective utilization of available resources namely Collaborative beamforming (CB) and cooperative transmission (CT). 
Lifetime of the network can be maximized by reducing the amount of data, RREQ packets towards the Sensing Point whose battery power is less than critical threshold. By utilizing energy of Sensing points which are nearby and then doing multi hop transmission can help in increasing the remaining energy [8].

The energy holes occur in the network when the battery of the Sensing point becomes critically ill. The existing methods which make use of either static sink or mobile sink ignore the concept of load balancing in the network which leads to reduce network lifetime.

When the data collection cycle triggers the mobile sink, Sensing points have to visit the target sites and collect the data, instead sensor Sensing points can transmit the data if the sink is within communication range which can improve the lifetime ratio of the network. The path of mobile sink can be reduced and flexible design can be provided so that either sensor can transmit or mobile sink can collect the data [9]. There is an overlap between region of interest and sensing area which needs to be significantly analysed to exponential increase the lifetime ratio. Each network has a set of configurations designated for specific applications and set of parameters can be auto-selected with the help of load balancing methods. The speed of parameter tuning and efficiency of implementation can be improved by making use of genetic algorithm as a plugin. [10].

When the sensors are connected to an Internet of Things (IoT) platform the efficiency for analysis of data produced by Sensing points increases. Few applications have wireless connection to IoT platform and few of them make use of wired connection.

The System of sensor Sensing points then communicate towards the gateway Sensing Point, which is then transmitted towards the internet server. The lifetime ratio of the network can be improved by making use of topology control methods which can maintain quality of communication links and also reduce the amount of packets dropped [11].

Wireless Sensor Sensing points are of smaller size and also have resource based constraints. When the Source Sensing Point loses its energy the position information of WSN changes after certain time. The method makes use of dynamic data of Sensing points along with route healing method to complete the path to increase lifetime ratio [12].

One of characteristics for the Sensing Point is its battery feature which effects the energy consumption. Multiple parameters like Sensing Point size, mobile nature, amount of coverage and connections to the Sensing Point also have an impact on lifetime ratio. The scheduling based on backbone is helpful in reducing the energy consumption along with balance of energy consumption to improve lifetime ratio [13].

The System of Sensing points is performed on Wireless Sensor Network (WSN) Sensing points. The method will make use of combination of packet delivery ratio along with lifetime ratio so that an optimized Sensing Point is elected for forwarding the data [14].

The entire area of the network is monitored to achieve better coverage. Cost and number of sensors are directly proportional. The placement of the Sensing points at correct positions will reduce energy consumption and increase lifetime ratio. Different shapes of Sensing Point placement are triangle, square and hexagon [15].

The WSN Sensing points will perform various tasks like temperature, weight computation, sending packets in the network region. WSN are adapted by military to track line of control as well as employees on the projects. The amount of cost for a WSN depends on the complex nature of network along with network size [16].

Wireless Sensor Network (WSN) contains a System of tiny sensors which are responsible for data Sensing as well as transmission to other Sensing points. When the amount of data receiving is considered more data is received by sensor Sensing points which are lesser distance to the control centre. Wireless Sensor Network (WSN) contains a System of tiny sensors which are responsible for data Sensing as well as transmission to other Sensing points. When the amount of data receiving is considered more data is received by sensor Sensing points which are lesser distance to the control centre. When the power is distributed in a non-uniform fashion then there is a creation of energy hole issue. Queue based approach is used to increase the lifetime ratio of the network based on power saving techniques [17].

Wireless Communication allows different devices to communicate with each other using infrastructure based protocol. Energy reduction is a major issue which is responsible for creation of holes in the network. The energy efficiency can be improved by making use of better connection between Sensing points [18].

Wireless sensor networks (WSNs) is used to detect the various parameters and have limited battery energy. The crucial factor is to save the energy which in turn increases lifetime ratio. The Sensing points whose distance is lesser with respect to sink Sensing Point they have more energy consumption and leads to lowest lifetime ratio [19].

The communication of sensor Sensing points with respect to sink happens with multi hop. The Sensing points which are near to the sink lost their energy levels. Hierarchical Least-Mean-Square (HLMS) makes use of adaptively filter which is adjusted based on error during packet deliver and filtering of Sensing points [20].

\section{GAME THEORY ALGORITHMS}

\section{A. Random Algorithm}

The random algorithm establishes the path between initiator to the control centre. When the initiator Sensing Point detects an information, it first finds the 1-hop Sensing points, send the request packet to all the 1-hop Sensing points, picks one the Sensing Point with respect to optimized time and then repeats process until path is established. The Random Algorithm can be summarized as provided in Fig. 4.

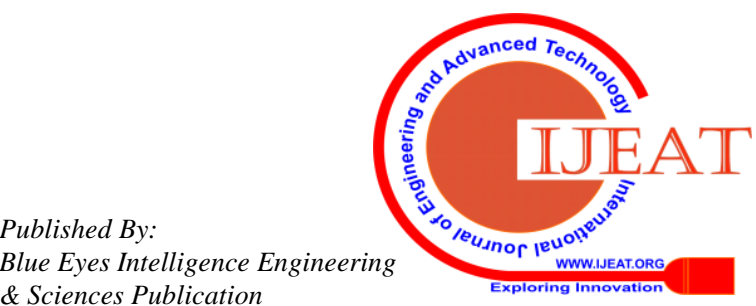




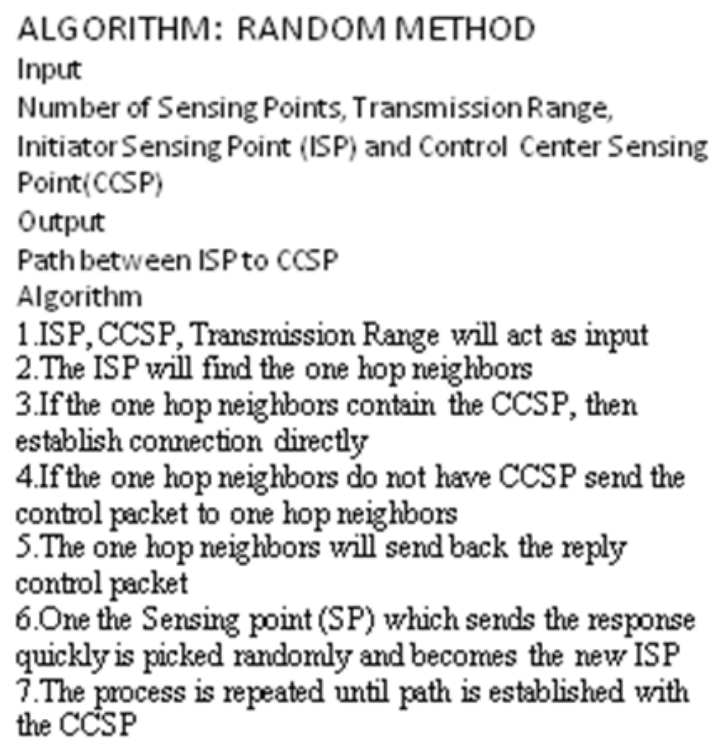

Fig. 4: Random Method

\section{B. Random-CGT}

This method will improve the selection of Sensing points based on computation of payoff. The payoff contains information about amount of packets in the present Sensing Point along with the communication disturbance which can happen. The entire area around the initiator Sensing Point is divided into multiple sections. For the packet to move forward a specific section is selected randomly, For the Sensing points in the selected section the payoff is computed, after that a Sensing Point which has the highest token is chosen as the next Sensing Point. Like this process is repeated until complete path is established. The token for the Sensing points is computed using the following formula

$$
\begin{aligned}
& T\left[U_{i}\right]=p\left[(-\Delta 1-\delta 1)\left(1-(1-q 1)^{N_{p n}-1}\right)+(v 1-\delta 1)(1-q 1)^{N_{p n}-1}\right] \\
& \text { Where, } \\
& \begin{array}{l}
q 1=\text { probability that the node will be next forward node } \\
\Delta 1=2 \delta 1 \\
\delta 1=51 \\
N_{p n}=\text { Number of player nodes } \\
v 1=6.15 \delta 1
\end{array}
\end{aligned}
$$

The Random-CGT algorithm can be summarized as shown in Fig 5.

Fig. 5: Random-CGT

\section{EGT-Random}

EGT-Random is used to find the optimized section and then picks a Sensing Point randomly. The initiator Sensing Point will find 1-hop Sensing points and then section is elected, forward Sensing Point is chosen randomly and then final path is established between initiator Sensing Point to destination control centre Sensing Point. The EGT-Random algorithm is summarized Fig. 6.

\section{ALGORITHM: RANDOM CGT Input}

Number of Sensing Points (SPs), Transmission Range, ISP and CCSP

$$
\text { Output }
$$

Path between ISP to CCSP Algorithm

1. ISP, CCSP, Transmission Range will act as input

2. The region around the ISP is divided into multiple sections

3. If the CCSP is within the transmission range, then stop

4. One of the section is chosen randomly

5. The payoff for all SPs are computed

6. The SP which corresponds to highest token is selected

7. The process is repeated until CCSP is found

\section{ALGORITHM: EGT-RANDOM} Input

Number of Sensing Points(SPs), Transm ission Range, ISP andCCSP Output

Path between ISP to CCSP Algorithm

1.ISP, CCSP, Transmission Range will act asinput

2.The region around the ISP is divided into multiple sections

3.If the CCSP is within the transmission range the com m unicate with CCSP and stop

4.The sector continuous function is com puted for multiple sections

5.Find the equilibrium for both the sections 6.The Ja cobean matrix is found

7.Eigen values are com puted for the Jacobean m atrix

8.The highest Eigen value is found and then the section which corresponds to the highest chosen as a forward section

9.After a section is selected then the SP in that section is picked up randomly

10.The process is repeated until CCSP is found

\section{Fig. 6: EGT-Random}

The sector continuous function is computed is defined in equation 2

$C_{k}=2 N_{k} E_{t r}-E_{t x}$

Where,

$N_{k}=$ Compute the Number of Player nodes

$E_{t r}=$ Energy required for recieving packet

$E_{t x}=$ Energy required for transmitting packet 
(2)

The energy consumed for receiving data is defined as below $E_{t r}(m)=m^{*} e_{r c}$

Where,

$m=$ Number of bits

$e_{r c}=$ transciever effectiven ess

The energy spend for transmission of data is defined in equation (4)

$E_{t x}(m, d)=m *\left(e_{t c}+e_{t a} * d^{\alpha}\right)$

Where,

$e_{t c}=$

energy spend by transmitter electronics

$e_{t a}=$

energy spend by transmitting amplifier

$d=$

dis tance between two nodes

$\alpha=$

path loss exp onent

The equilibrium equation can be defined as below

$E Q_{1}=\frac{E_{1}-E_{2}+\lambda C_{2}}{\lambda\left(C_{1}+C_{2}\right)}$

$E Q_{2}=1-E Q_{1}$

The Jacobian Matrix is computed as below

$J C\left(X_{1}, X_{2}\right)=\left[\begin{array}{cc}-\lambda \beta 1 C F_{1} E Q_{1} E Q_{2} & \lambda \beta 1 C F_{2} E E_{1} Q E Q_{2} \\ \lambda \beta 1 C F_{1} E Q_{1} E Q_{2} & -\lambda \beta 1 C F_{2} E Q_{1} E Q_{2}\end{array}\right] \ldots \ldots \ldots$

\section{GTEB Algorithm}

The GTEB Algorithm uses a combination of Random-CGT and EGT-Random. The EGT-Random is the method which is responsible for selection of a particular section in the transmission range in an optimized way and Random-CGT selects an optimized Sensing Point based on value of payoff. GTEB takes the advantages of both the method and provides an optimized path which is energy efficient. The GTEB Method is summarized in Fig. 7.

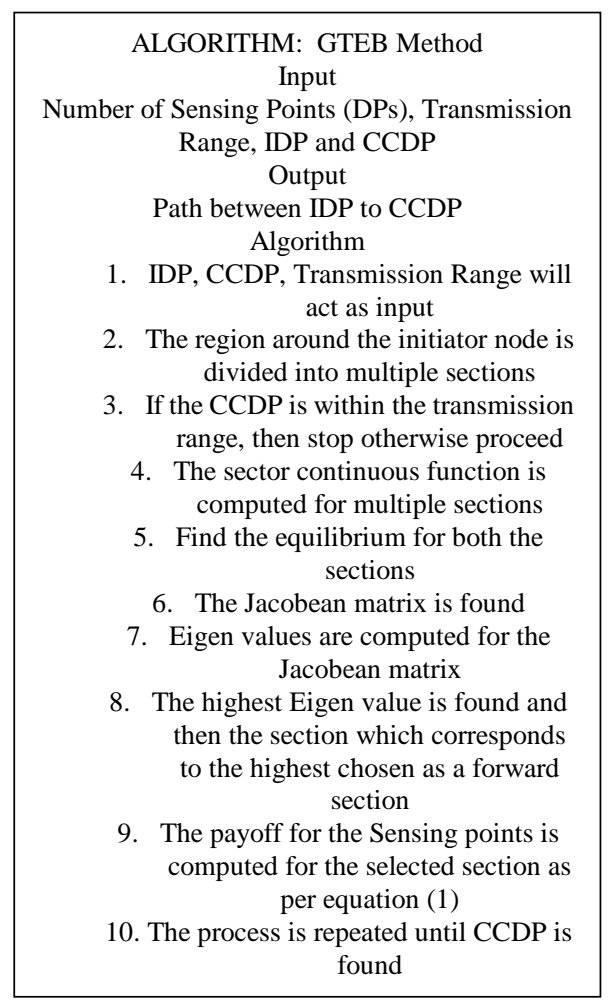

Fig. 7: GTEB Method

\section{RESULTS AND DISCUSSION}

This section provides the information about the simulation results obtained by MATLAB programming for game theory algorithms

\section{A. GTEB Results}

The simulation Set Up for GTEB Method is defined as below Table1: GTEB Simulation Input

\begin{tabular}{|l|l|}
\hline Parameter & Parameter Value \\
\hline Number of Sensing points & 100 \\
\hline Range for Direct Communication & $40 \mathrm{~m}$ \\
\hline Transmission Energy & $15 \mathrm{~mJ}$ \\
\hline Generation Energy & $5 \mathrm{~mJ}$ \\
\hline Area & $100 * 100$ \\
\hline Attenuation Factor & 0.5 \\
\hline Initiator Sensing Point & 6 \\
\hline Destination Control Centre & 23 \\
\hline Initial Energy & $2000 \mathrm{~J}$ \\
\hline
\end{tabular}




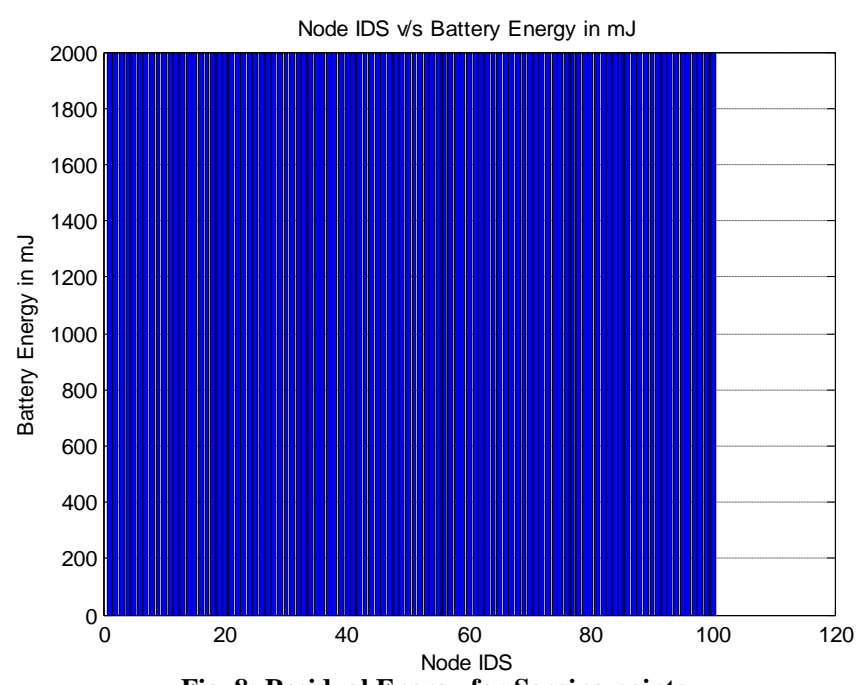

Fig. 8: Residual Energy for Sensing points

Fig. 8 shows the remaining energy for Sensing points in the network. As shown in the Fig. 8 all the 100 Sensing points have been initialized with the same amount of energy levels of 2000J.

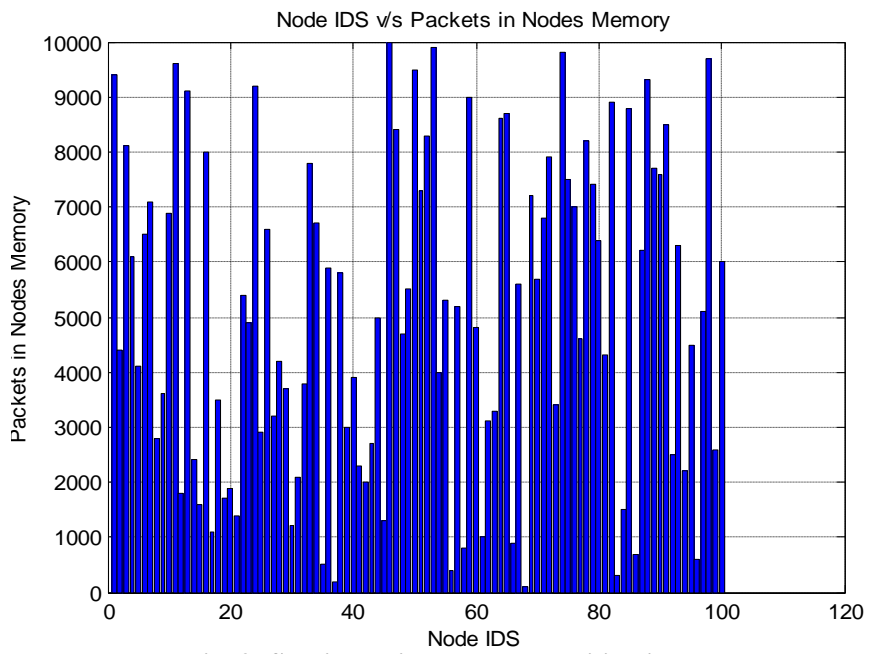

Fig. 9: Sensing points Memory Utilization

Fig. 9 shows the Sensing points memory utilization. As shown in the Fig. 9. Sensing Point 1 has the remaining energy of 9300 packets remaining in its memory and in a similar fashion different Sensing points have different energy levels.

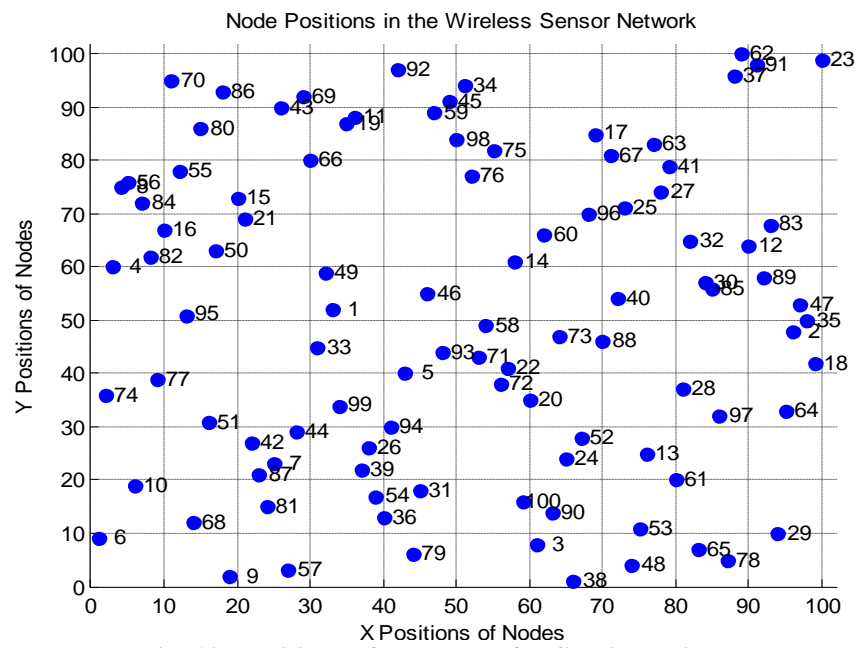

Fig. 10: Positional Occurrence for Sensing points
Fig. 10 shows the position of the various Sensing points in the network which are spread in an area of $100 * 100 \mathrm{~m}$. As shown in the Fig. 10 each Sensing Point has its own unique position in the network. Sensing Point6 is placed at the coordinate $(2,9)$. Sensing Point 20 is placed at the coordinate $(60,35)$. In a similar fashion all other Sensing points have the unique value of position.

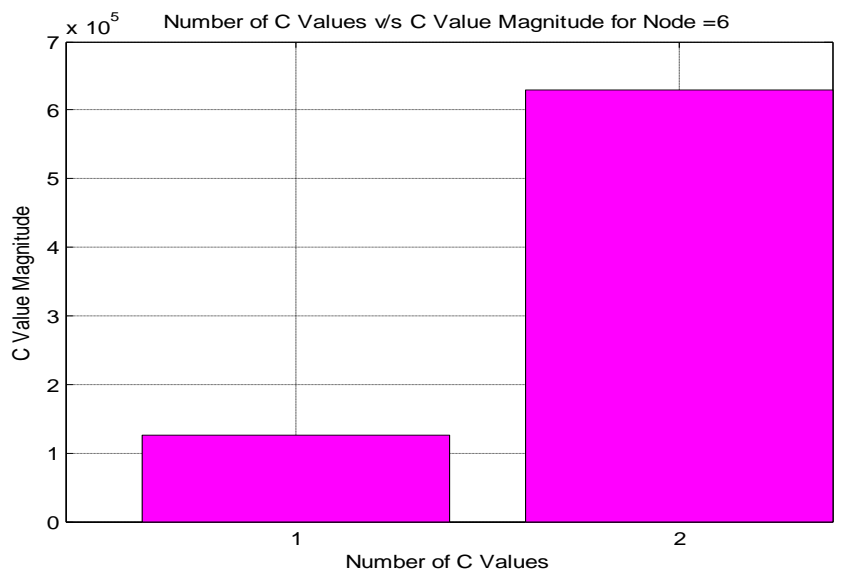

Fig. 11: Equilibrium Equation

Fig. 11shows the equilibrium equation. As shown in the Fig. 11 section 1 has the value of $0.1 \mathrm{M}$ and for the section2 the value is $0.62 \mathrm{M}$.

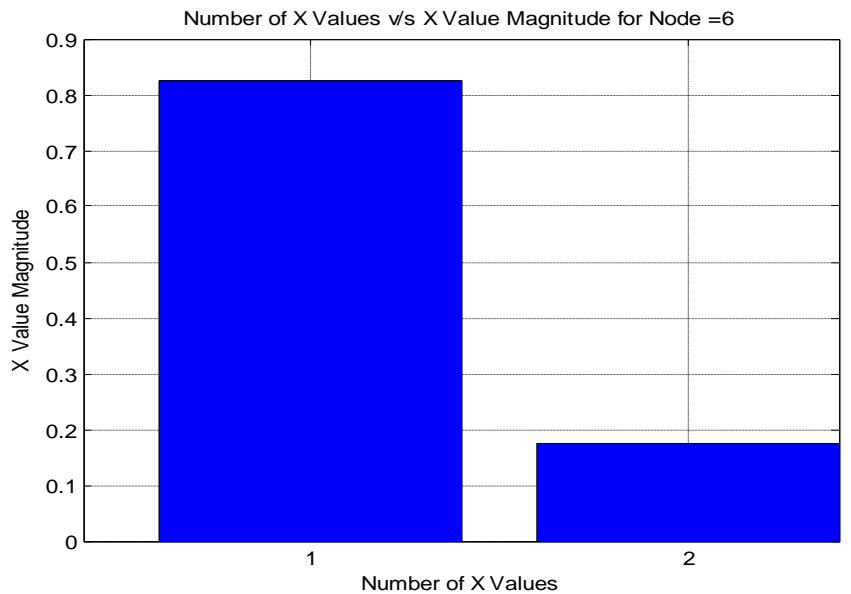

Fig. 12: Equilibrium Values for Section

Fig. 12 shows the equilibrium values for the sections. As shown in the Fig. 120.82 is the equilibrium value for section 1 and 0.18 is the equilibrium value for section 2 .

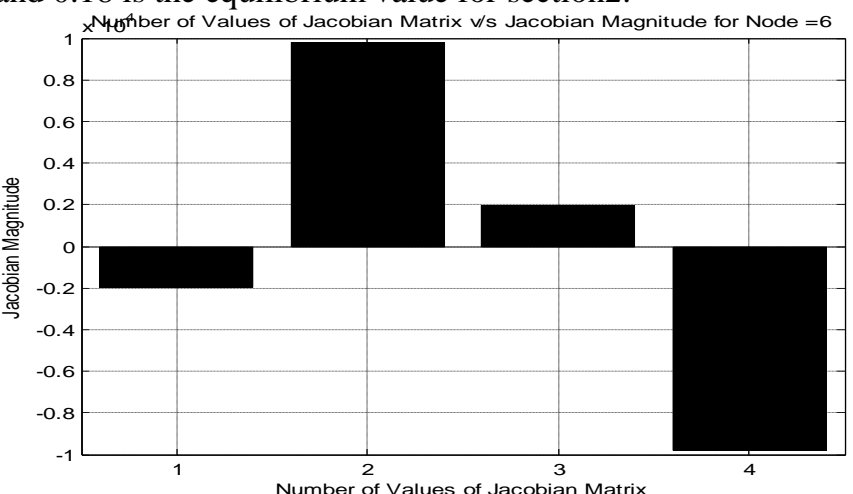

Fig. 13: Jacobian Matrix 
Fig. 13 shows the Jacobian Matrix. The four values as shown in the Fig. 13 are $-0.2,0.8,0.2$ and -0.8 . The Jacobean has the significance of equal magnitude and opposite direction with 0.2 and 0,8 as distinct values and having opposite direction of +- .

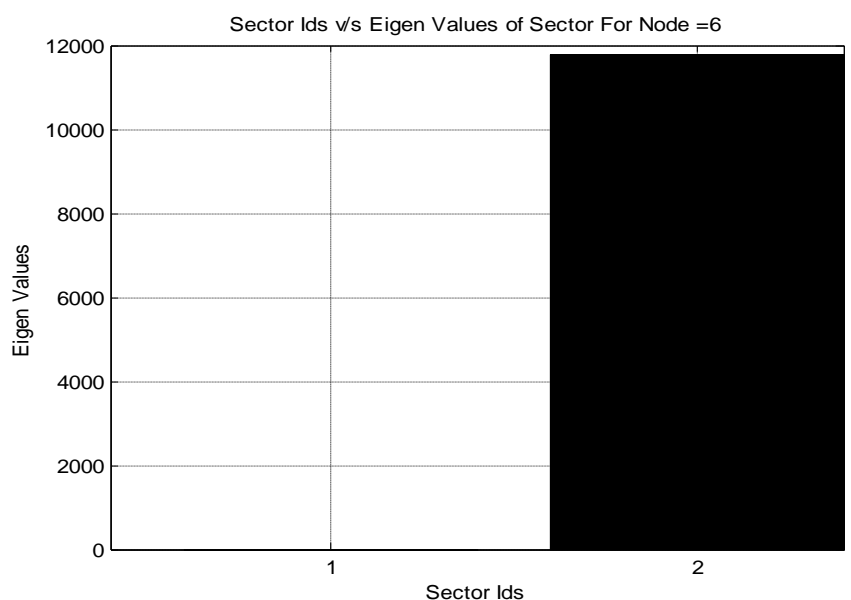

Fig. 14: Eigen Value Computation for Sections

Fig. 14 shows the Eigen value computation for sections.

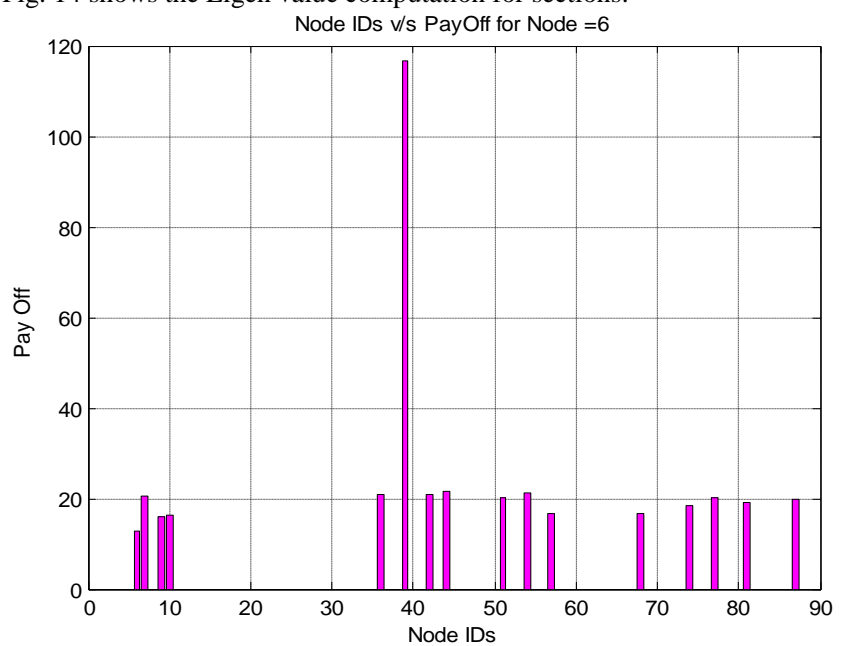

Fig. 15: Pay Off Measure

Fig. 15 shows the payoff computation. The payoff is visible only for the Sensing points which are within transmission range of Sensing Point6 which is initiator Sensing Point. Sensing Point 8 has the payoff of about 15 , Sensing Point 40 has the payoff of about 110 .

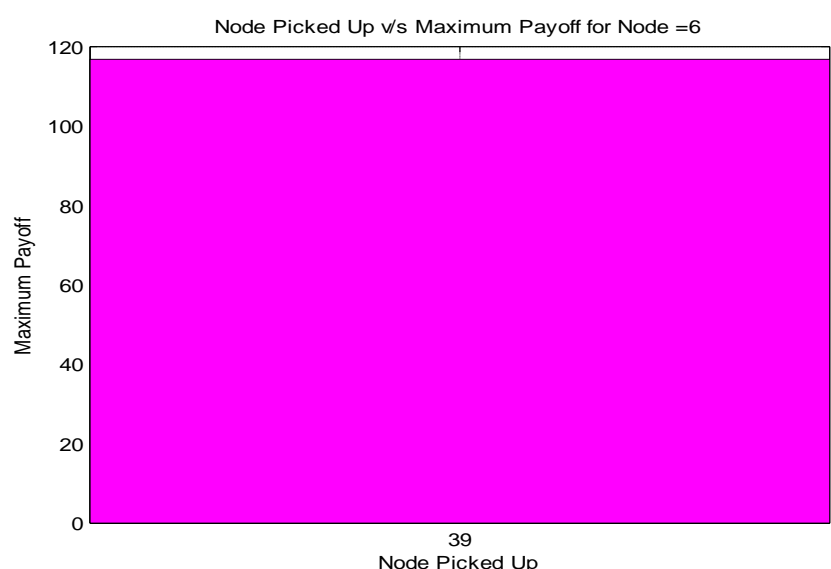

Fig. 16: Forward Sensing Point Selection
Fig. 16 shows the forward Sensing Point which is selected in Sensing Point 39 because it is having the highest value of payoff which is around 118 .

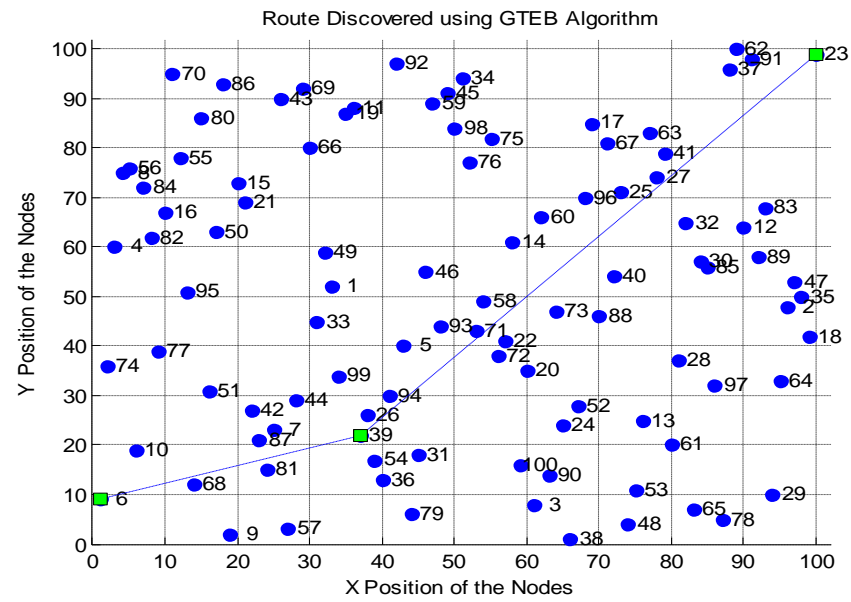

Fig. 17: Path for GTEB Method

Fig. 17 shows the path for GTEB Method. As shown in the Fig. 17 Sensing Point 6 sends the transmission to Sensing Point39 and then Sensing Point39 communicates to Sensing Point 23.

\section{B. Comparison Results}

The comparison between GTEB, Random-EGT, CGT-Random and Random method is performed on the basis of the following simulation setup.

Table2: Comparison Simulation Input

\begin{tabular}{|l|l|}
\hline Name of Attribute & Value \\
\hline Number of Sensing points & 200 \\
\hline Range for Direct Communication & $80 \mathrm{~m}$ \\
\hline Energy for Link Formation & $20 \mathrm{~mJ}$ \\
\hline Energy for Control data generation & $10 \mathrm{~mJ}$ \\
\hline Area & $200 * 200$ \\
\hline Attenuation Factor & 0.5 \\
\hline Initiator Sensing Point & 105 \\
\hline Destination Control Centre & 63 \\
\hline Number of Iterations for Comparison & 50 \\
\hline Initial Energy & $3000 \mathrm{~J}$ \\
\hline
\end{tabular}

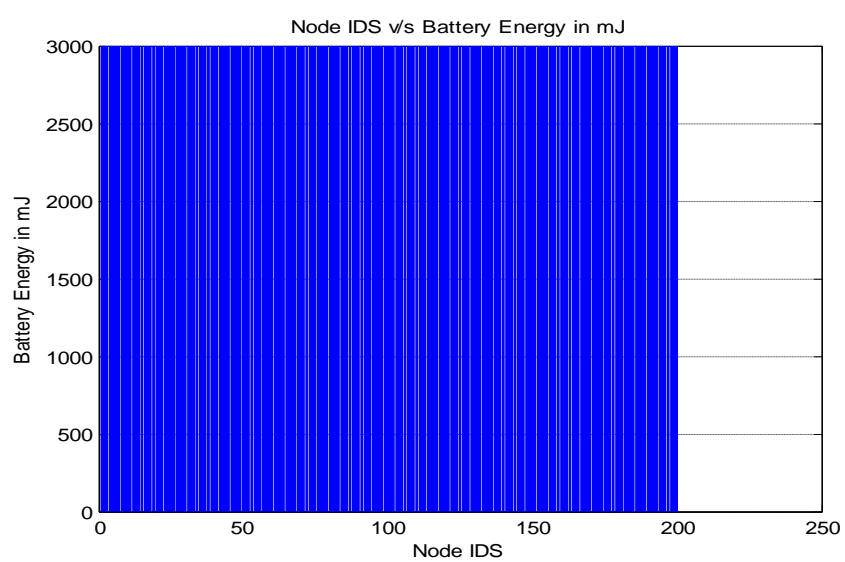

Fig. 18: Energy for Sensing points when network is formed 
Fig. 18 shows the energy for the Sensing points in the network. 200 Sensing points in the network have been given an energy of $3000 \mathrm{~J}$. The $\mathrm{x}$-axis is the number of Sensing points in the network and the y-axis is the indication for energy for the Sensing points in the network.

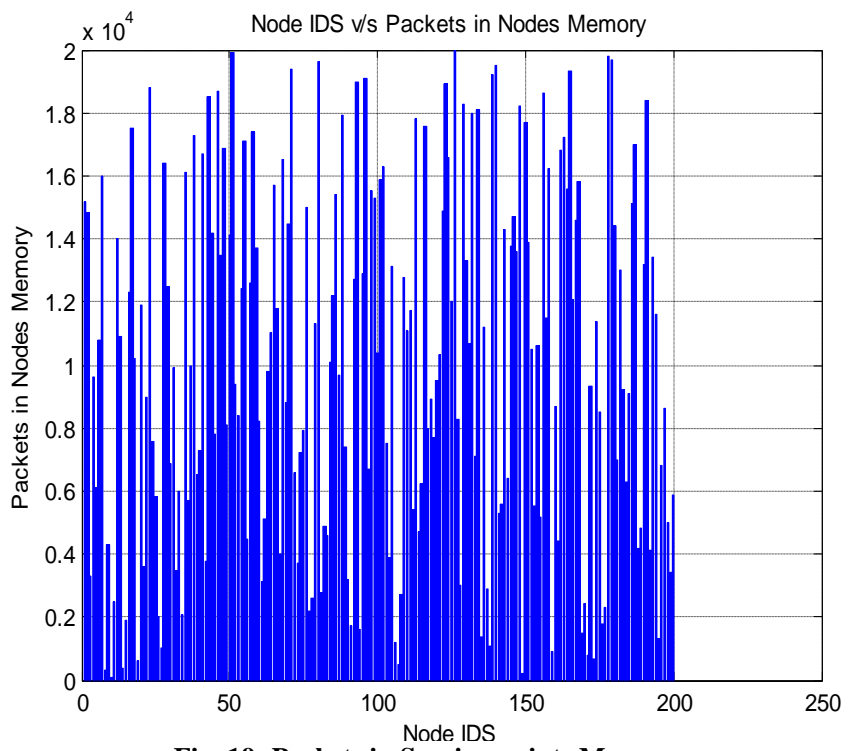

Fig. 19: Packets in Sensing points Memory

Fig. 19 shows the packets present in the Sensing points memory. As shown in the fig the $\mathrm{X}$-axis indicates the number of Sensing points in the network and Y-axis is the packets present in the Sensing points memory. As shown in the Fig. 19 Sensing Point 1 has $145 \mathrm{~kb}$ of packets and then Sensing Point 200 has $60 \mathrm{~kb}$ of packets. In a similar fashion each and every of 200 Sensing points have there own packet distribution.

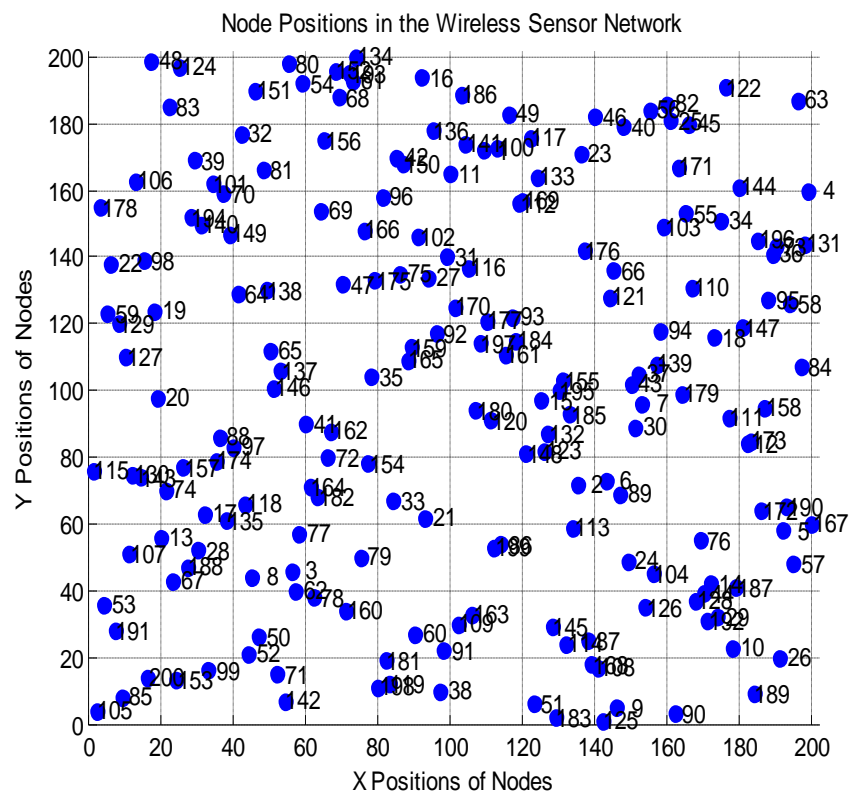

Fig. 20: Position for Sensing points in Network

Fig. 20 has the representation for position of Sensing points in the network. As shown in the Fig. 20 there are 200 Sensing points spread across an area of 200* $200 \mathrm{~m}$. Sensing Point 91 is at a position of $(15,24)$. Sensing Point 42 is at a position of $(50,10)$, All the remaining sensing points have their own multidimensional value.

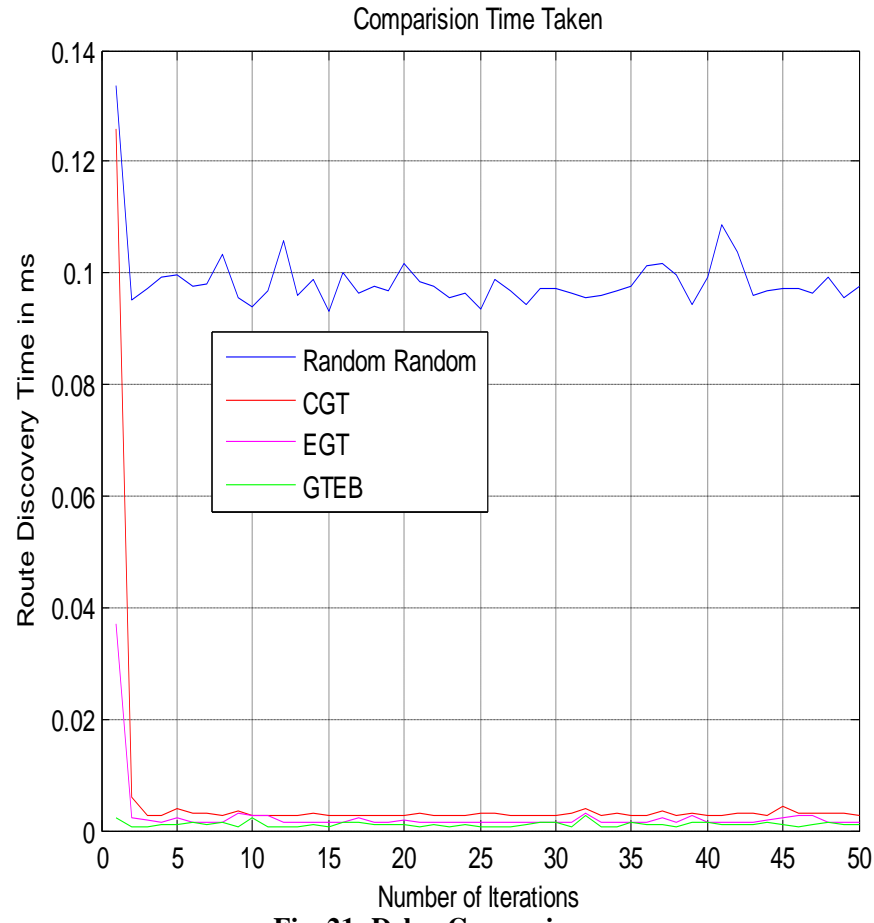

Fig. 21: Delay Comparison

Fig. 21 shows the control packet time consumption to establish link of all Sensing points in the path for sending packets. GTEB has the lowest delay compared CGT, EGT and Random algorithms. The delay of GTEB method is not exceeding 0.005 in any case. In a same way the delay of EGT and CGT never exceeds $0.07 \mathrm{~ms}$ and random method has the delay which is above $0.08 \mathrm{~ms}$.

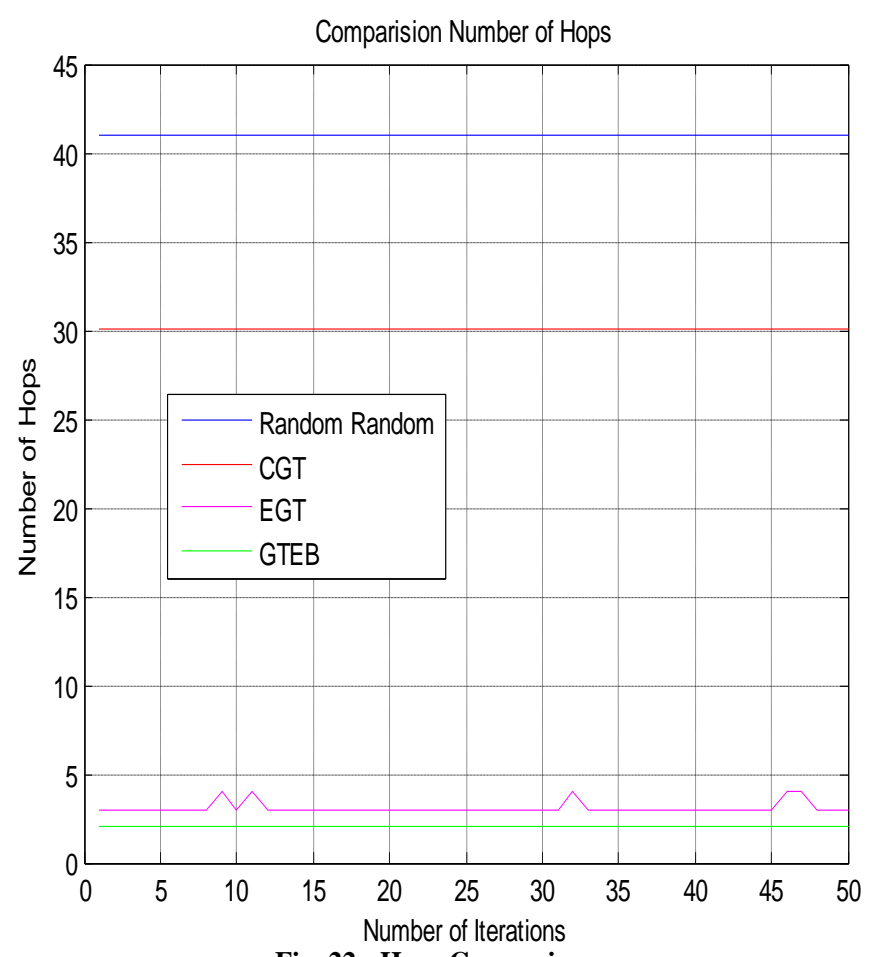

Fig. 22: Hops Comparison

Fig. 22 shows the hops comparison. As shown in the Fig. 22 GTEB algorithm has the lowest number of hops of about 4 followed by EGT. CGT and Random method. 


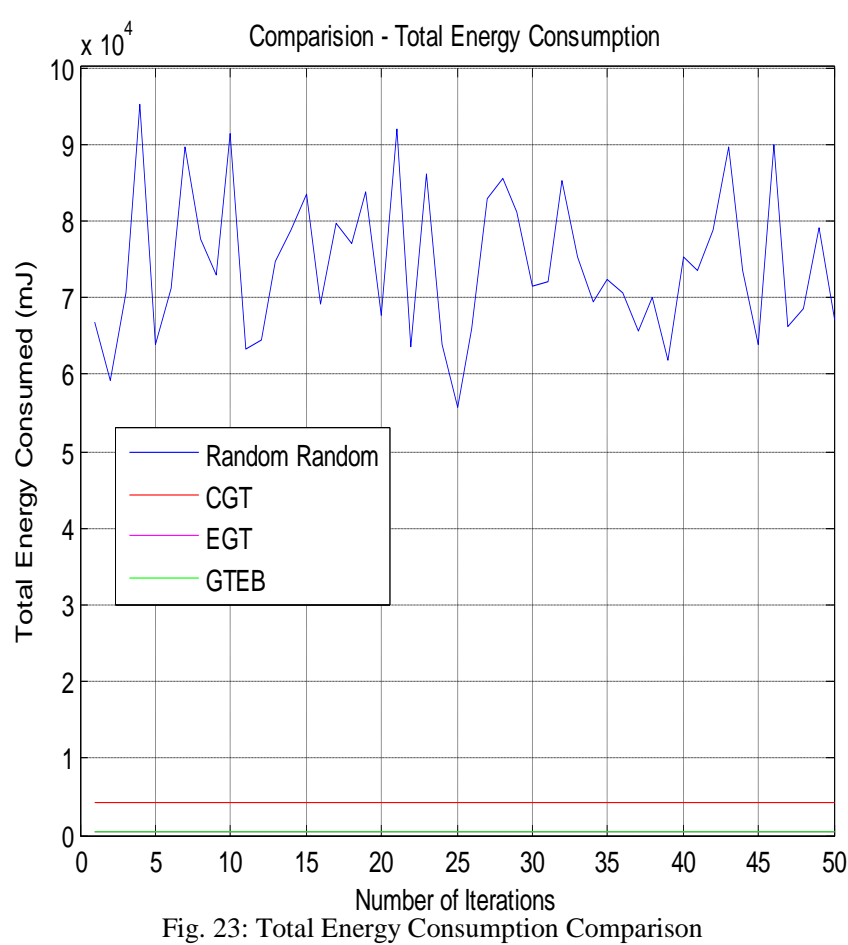

Fig. 23 shows the comparison of total energy consumption. As shown in the Fig. 23 GTEB method has the lower energy consumption followed by CGT, EGT and Radom has the highest energy consumption. $0.1 \mathrm{~kJ}$ is the total energy consumed for GTEB across multiple iterations followed by CGT and EGT which is around $0.3 \mathrm{~kJ}$ and random is around $0.6 \mathrm{~kJ}$ to $0.8 \mathrm{~kJ}$ range.

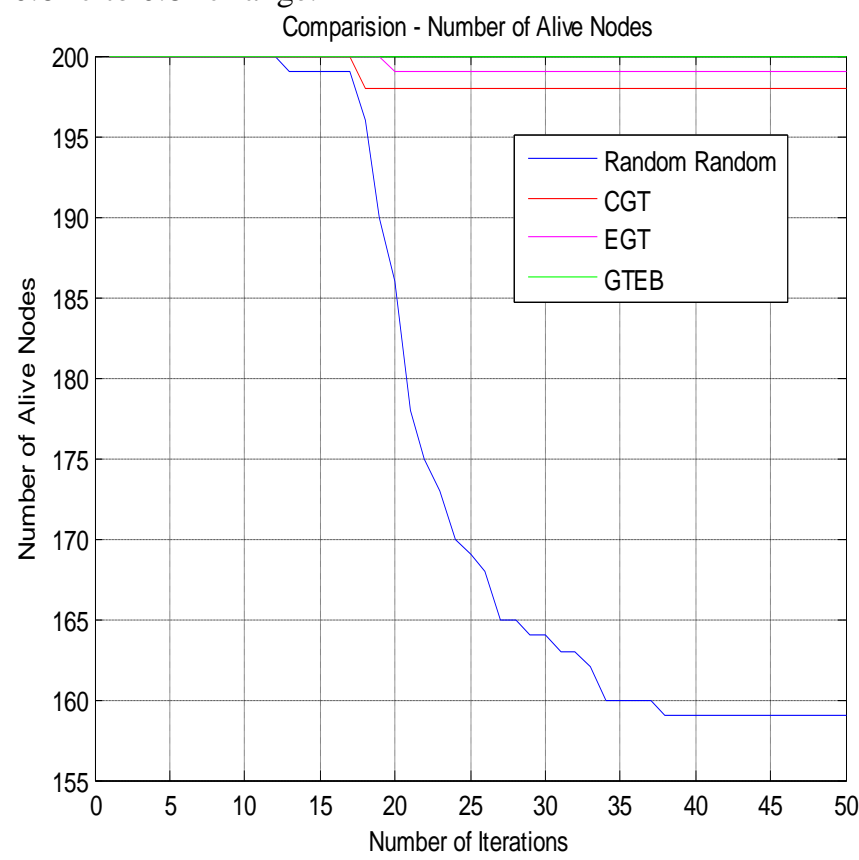

Fig. 24: Hole Sensing Points Comparison

Fig. 24 shows the comparison of Hole Sensing points. As shown in the Fig. 24 for GTEB method has the highest number of Hole Sensing points followed by EGT, CGT and Random. At the end of 50 iterations the GTEB algorithm is having 200 Hole Sensing points followed by EGT which is having 198 Hole Sensing points followed by CGT which is having 196 Hole and Radom algorithm has 158 Hole Sensing points. Also as the number of iterations increases the Hole Sensing points reduces.

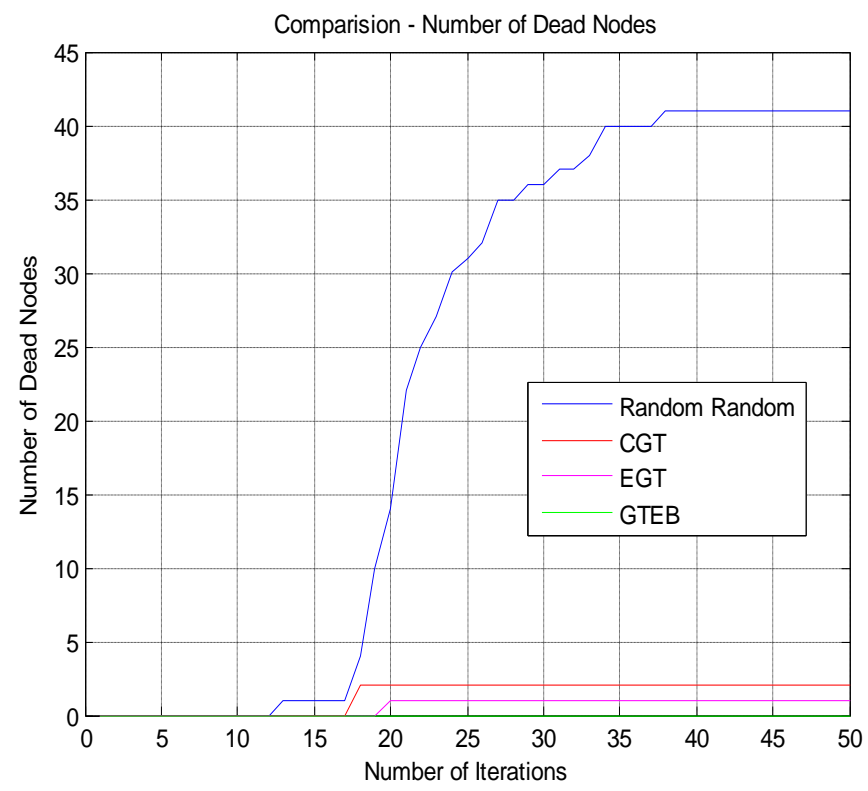

Fig. 25: Non-Hole Sensing Points Comparison

Fig. 25 shows the number of Non-Hole Sensing points. GTEB has lowest number of Non-Hole Sensing points followed by EGT, CGT and Random. As shown in the Fig. 25 at the end of 50 iterations GTEB has 0 Non-Hole Sensing points followed by EGT which is having 2 Non-Hole Sensing points, CGT has 4 Non-Hole Sensing points and Random method has 42 Non-Hole Sensing points.

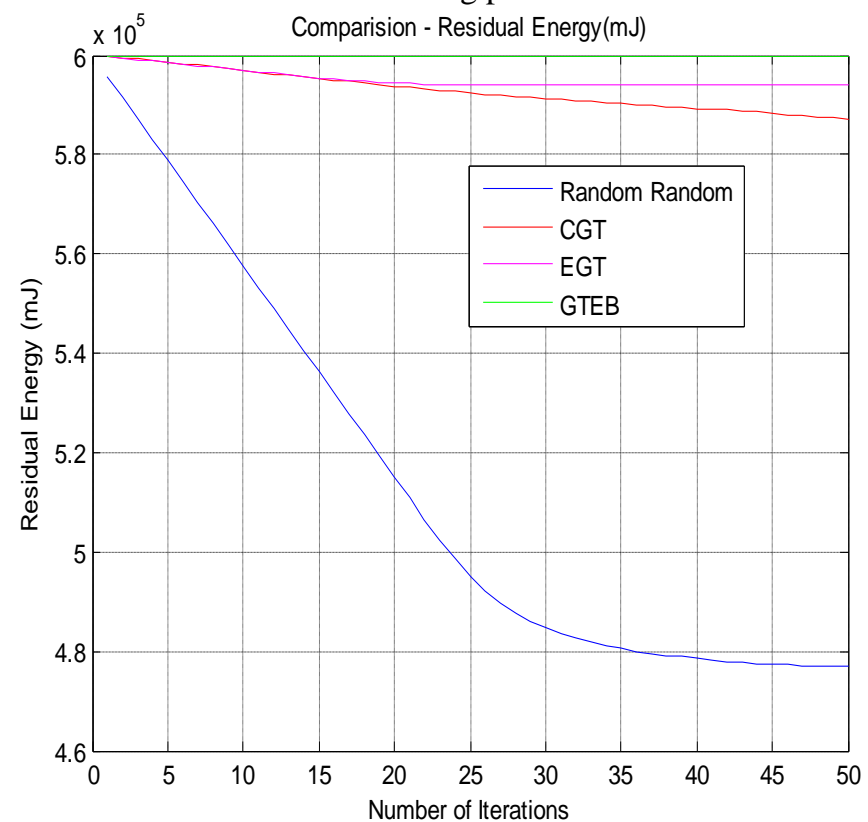

Fig. 26: Residual Energy Comparison

Fig. 26 shows the residual energy for the network. GTEB has highest residual energy followed by EGT, CGT and least residual energy is of Random method. At the end of 50 iterations GTEB has $0.6 \mathrm{MJ}$ of residual energy followed by EGT which has the residual energy of $0.585 \mathrm{MJ}$, followed by CGT which has the residual energy of about $0.583 \mathrm{MJ}$ and least is of random method which has the residual energy of about $0.467 \mathrm{MJ}$. 


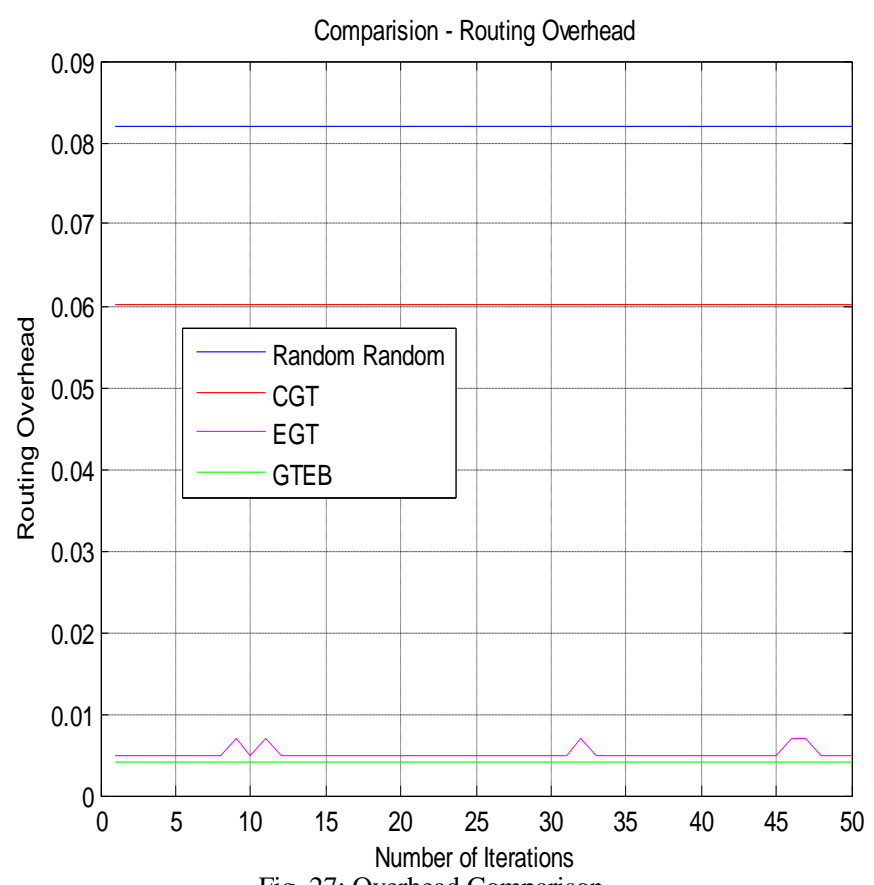

Fig. 27: Overhead Comparison

Fig. 27 shows the overhead factor comparison. GTEB has the lowest overhead followed by EGT, CGT and Random Method. Also the overhead of GTEB never exceeds 0.005 and that of EGT never exceeds 0.008, CGT never exceeds 0.06 and random never exceeds 0.085 .

\section{V.CONCLUSION}

The paper discusses about kinds of WSN network, its applications, review of energy efficient and lifetime ratio improvement techniques with the help of game theory methods. Few game theory algorithms namely Random, Random CGT, EGT Random and GTEB method are discussed in detail. The algorithms are also simulated in MATLAB. From the simulation results one can prove that the GTEB method outperforms Radom CGT, EGT Random and Random methods with respect various factors.

\section{REFERENCES}

1. M. Abd, S. F. Majed Ai Rubeaai, K. Tepe and R. Benlamri, "Game theoretic energy balancing routing in three dimensional wireless sensor networks," 2015 IEEE Wireless Communications and Networking Conference (WCNC), New Orleans, LA, 2015, pp. 1596-1601.

2. Mehmmood A. Abd ; Brajendra K. Singh ; Sarab F. Al Rubeaai ; Kemal E. Tepe ; Rachid Benlamri, "Game theoretic energy balanced (GTEB) routing protocol for wireless sensor networks", 2014 IEEE Wireless Communications and Networking Conference (WCNC),6-9 April 2014

3. R. Kumar and S. Kad, "Improved Wireless Sensor Network Lifetime by Fuzzy Logic Based Dynamic Clustering with Global Energy Balance," 2017 International Conference on Next Generation Computing and Information Systems (ICNGCIS), Jammu, 2017, pp. 104-109.

4. A. Vishwanath, R. Datta and N. Marchang, "Probabilistic Sensing Point Scheduling in Dense Wireless Sensor Networks," TENCON 2019 - 2019 IEEE Region 10 Conference (TENCON), Kochi, India, 2019, pp. 1169-1173.

5. H. Wang and H. Chen, "On the Construction of Data Aggregation Tree with Maximized Lifetime in Wireless Sensor Networks," 2019 11th International Conference on Wireless Communications and Signal Processing (WCSP), Xi'an, China, 2019, pp. 1-6

6. H. Wang and H. Chen, "On the Construction of Data Aggregation Tree with Maximized Lifetime in Wireless Sensor Networks," 2019 11th International Conference on Wireless Communications and Signal Processing (WCSP), Xi'an, China, 2019, pp. 1-6.
7. G. Saad, H. Harb, C. A. Jaoude and A. Jaber, "A Distributed Round-Based Prediction Model for Hierarchical Large-Scale Sensor Networks," 2019 International Conference on Wireless and Mobile Computing, Networking and Communications (WiMob), Barcelona, Spain, 2019, pp. 1-6.

8. Y. Dong, J. Wang, B. Shim, and D. I. Kim, "DEARER: A distanceand-energy-aware routing with energy reservation for energy harvesting wireless sensor networks," IEEE J. Sel. Areas Commun., vol. 34, no. 12, pp. 3798-3813, Dec, 2016.

9. X. He, X. Fu and Y. Yang, "Energy-Efficient Trajectory Planning Algorithm Based on Multi-Objective PSO for the Mobile Sink in Wireless Sensor Networks," in IEEE Access, vol. 7, pp. 176204-176217, 2019.

10. L. Brisolara, M. Lorenzatto Braga, A. Braga and P. Roberto Ferreira, "Parameter Tuning in Load Balancing Techniques for Wireless Sensor Networks through Genetic Algorithms," 2019 8th Brazilian Conference on Intelligent Systems (BRACIS), Salvador, Brazil, 2019 , pp. $42-47$.

11. T. N. Nguyen, C. V. Ho and T. T. T. Le, "A Topology Control Algorithm in Wireless Sensor Networks for IoT-based Applications," 2019 International Symposium on Electrical and Electronics Engineering (ISEE), Ho Chi Minh, Vietnam, 2019, pp. 141-145.

12. C. B. N. Lakshmi and S. K. Mohan Rao, "Bio-inspired self-healing routing to improve lifetime of wireless sensor networks," 2014 International Conference on Communication and Network Technologies, Sivakasi, 2014, pp. 134-138.

13. D. Sharmila, R. Sujitha and G. Rajkumar, "On improving the lifetime of wireless sensor networks using virtual scheduling backbone replacement," 2013 IEEE Conference on Information \& Communication Technologies, Thuckalay, Tamil Nadu, India, 2013 pp. 249-252.

14. M. S. Manshahia, M. Dave and S. B. Singh, "Firefly algorithm based clustering technique for Wireless Sensor Networks," 2016 International Conference on Wireless Communications, Signal Processing and Networking (WiSPNET), Chennai, 2016, pp. 1273-1276.

15. N. Gupta, M. Wazid, S. Sharma, D. P. Singh and R. H. Goudar, "Coverage life time improvement in Wireless Sensor Networks by novel deployment technique," 2013 IEEE International Conference ON Emerging Trends in Computing, Communication and Nanotechnology (ICECCN), Tirunelveli, 2013, pp. 293-297.

16. M. Singh and P. Gill, "Wireless sensor networks with effective algorithm for long life and energy optimization," 2017 International Conference on Electrical, Electronics, Communication, Computer, and Optimization Techniques (ICEECCOT), Mysuru, 2017, pp. 32-34

17. F. Jiang, H. Wu, D. Huang and C. Lin, "Lifetime Security Improvement in Wireless Sensor Network Using Queue-Based Techniques," 2010 International Conference on Broadband, Wireless Computing, Communication and Applications, Fukuoka, 2010, pp. 469-474.

18. M. Patil and C. Sharma, "Energy efficient cluster head selection to enhance network connectivity for wireless sensor network," 2016 IEEE International Conference on Recent Trends in Electronics, Information \& Communication Technology (RTEICT), Bangalore, 2016, pp. 175-179.

19. A. L. Kakhandki, S. Hublikar and P. Kumar, "An efficient transceiver optimization based routing technique for wireless sensor network," 2016 International Conference on Communication and Electronics Systems (ICCES), Coimbatore, 2016, pp. 1-5

20. L. Tan and M. Wu, "Data Reduction in Wireless Sensor Networks: A Hierarchical LMS Prediction Approach," in IEEE Sensors Journal, vol. 16, no. 6, pp. 1708-1715, March15, 2016. doi 10.1109/JSEN.2015.2504106 